\title{
Estimation quantitative de la caecotrophie chez le lapin en croissance : variations en fonction de l'âge
}

\author{
T. GIDENNE et F. LEBAS \\ INRA, Laboratoire de Recherches sur l'Elevage du Lapin \\ Centre de Recherches de Toulouse \\ B.P. 27, F 31326 Castanet Tolosan Cedex
}

\begin{abstract}
Résumé
Cette étude a pour but de mesurer la production de caecotrophes au cours de la croissance du Lapin, ainsi que d'estimer quantitativement la caecotrophie en fonction de paramètres zootechniques, simples à mesurer. Des lapines de race Néozélandais blanc $\times$ Californien, recevant $\mathrm{ad}$ libitum un aliment complet granulé, ont été élevées en cage à digestibilité de 25 jours à 133 jours d’âge. La mesure de la quantité de caecotrophes émise a été réalisée pendant $24 \mathrm{~h}$, chaque semaine jusqu'à 63 jours d'âge, puis toutes les deux semaines. A chaque stade, 7 à 10 individus, dont l'ingestion et l'excrétion n'ont pas été perturbées par le port du collier interdisant l'ingestion des caecotrophes, ont été retenus parmi les 10 à 15 ayant reçu ce collier.

La teneur en matière sèche (MS) et la quantité sèche de caecotrophes se stabilisent, après 11 semaines d'âge, respectivement entre 36 et $40 \%$, et aux environs de $20 \mathrm{~g}$ de MS/jour. L'excrétion totale fécale de MS (caecotrophes + fèces dures) exprimée en \% de l'ingéré alimentaire ne varie pas significativement en fonction de l'âge $(48$ à $55 \%)$. La teneur en matières azotées totales (MAT) des caecotrophes, double de celle des fèces dures, passe de 23 à $27 \%$ de la MS entre 63 et 133 jours d'âge, et l'apport de MAT par les caecotrophes atteint $26 \%$ de l'apport azoté alimentaire entre 8 à 9 semaines d'âge. La teneur en cellulose brute des caecotrophes est $50 \%$ plus basse (20 à $24 \%$ ) que celle des fèces dures, sans variations importantes en fonction de l'âge.

C'est en période de forte croissance que le recyclage de MS (et de MAT) par la pratique de la caecotrophie est maximum. De 28 à 63 jours d'âge, la production de caecotrophes est bien corrélée avec une combinaison linéaire de la consommation d'aliment et de l'excrété de fèces dures $(\mathrm{R}=0,827 ; \mathrm{P}<0,01)$. Ainsi, avant l'âge d'abattage (11 semaines) et pour notre régime expérimental, il semble possible d'estimer quantitativement la production de caecotrophes en évitant la pose d'un carcan, à partir de paramètres zootechniques aisément mesurables.
\end{abstract}

Mots clés : Lapin, caecotrophie, effet de l'âge, équations de prédiction.

\section{Introduction}

L'intérêt nutritionnel de la caecotrophie pour le Lapin a déjà été signalé par plusieurs auteurs, tant au plan de son apport azoté que vitaminique (BATTAGLINI, 1968 ; Kulwich et al., $1953 ; \ldots)$. Un travail de synthèse a d'ailleurs été réalisé à ce sujet par Gallouin en 1981. De plus, la pratique de la caecotrophie intervient sur le transit et la composition des digesta (PIekARz, 1963 ; Jilge, 1974 ; CATAla, 1976 ; Gidenne \& Lebas, $1984 ;. .$. .). Toutefois, peu d'études estiment quantitativement la production de caecotrophes, et ses variations en fonction de l'âge de l'animal ou de sa croissance. 
Or, nous avons montré, dans un travail antérieur (Gidenne \& PonceT, 1985) qu'il était nécessaire d'estimer quantitativement la caecotrophie pour le calcul de la digestibilité apparente des nutriments dans les différents segments digestifs du lapin. Nous avions alors décrit une technique d'estimation, relativement lourde, basée sur la distribution d'un aliment marqué à des lapins pouvant pratiquer librement la caecotrophie (absence de stress), associée à des abattages répartis sur 24 heures.

La présente étude a donc pour but de mesurer la production de caecotrophes en fonction de l'âge des animaux, mais à l'aide de la technique habituelle : la pose d'un collier pour empêcher l'ingestion des caecotrophes émis. De plus, à partir de ces mesures, nous essaierons de trouver des équations permettant de prédire la quantité de caecotrophes émise, en fonction de paramètres aisément mesurables, tels que la consommation d'aliment ou la quantité de fèces dures excrétées.

\section{Matériel et méthodes}

\section{A. Type d'animal et alimentation}

Des lapines métisses (Néozélandais blanc $\times$ Californien) ont été maintenues en cage à digestibilité, de 25 jours (sevrage) à 133 jours d'âge. Afin de limiter le stress du sevrage, les animaux ont été élevés avec deux frères ou sœurs durant les 3 premiers jours d'expérience. Ils ont été nourris ad libitum, avec un aliment à base de luzerne déshydratée $(40 \%)$, d'orge $(30 \%)$, d'avoine $(10 \%)$, de tourteaux de tournesol $(10 \%)$ et de soja $(5 \%)$, minéraux et vitamines $(5 \%)$. La teneur en matières azotées totales (MAT) du régime est de $16,5 \%$ de la matière sèche (MS), la teneur en NDF (Méthode de Van Soest) est de $34 \%$ de la MS et la teneur en cellulose brute, de $17,7 \%$ de la MS.

\section{B. Récolte des caecotrophes et contrôles zootechniques}

Chaque semaine jusqu'à l'âge de 63 jours, puis toutes les deux semaines jusqu'à l'âge de 133 jours, et pour une durée limitée à 24 heures, un collier empêchant l'ingestion des caecotrophes a été placé sur 10 à 15 lapins. Ces lapins mis en essai étaient représentatifs d'un groupe de 20 constitué au sevrage de manière à être certain de disposer d'assez d'animaux en fin d'essai. Deux groupes comparables de 20 individus ont été utilisés, l'un pour les semaines paires, l'autre pour les semaines impaires, de manière à assurer un délai d'au moins deux semaines entre deux poses successives de collier chez le même individu. Pour minimiser la période de stress, et éviter une modification trop importante du cycle digestif, la durée de la mesure a été fixée à $24 \mathrm{~h}$; les lapins ont ainsi porté un collier de $14 \mathrm{~h}$ à $14 \mathrm{~h}$ le lendemain. Toujours dans le but de limiter le stress, nous avons mis au point un collier aussi léger que possible, mais suffisamment rigide pour empêcher l'ingestion des caecotrophes. Le collier a été fabriqué à l'aide de PVC «cristal » légèrement souple (épaisseur : $0,11 \mathrm{~cm}$ ), dont le diamètre intérieur est adapté à la taille du cou de l'animal. Ainsi, le poids du collier est de $17 \mathrm{~g}$ (diamètre extérieur : $13 \mathrm{~cm}$ ) pour des animaux âgés de 4 semaines; il atteint 60 à $70 \mathrm{~g}$ (diamètre extérieur : $26 \mathrm{~cm}$ ) chez l'adulte (environ $1,5 \%$ du poids vif). 
Le poids vif des animaux, ainsi que leur consommation d'aliment ont été mesurés chaque semaine. Les quantités de fèces dures excrétées et d'aliment ingérées ont, en outre, été contrôlées le jour de la récolte des caecotrophes, ainsi que la veille et le lendemain.

\section{Analyses chimiques et statistiques}

La teneur en matière sèche (MS) des fèces dures et des caecotrophes a été déterminée par dessication, 48 heures à $80^{\circ} \mathrm{C}$, pour chaque échantillon récolté. Les teneurs en matières azotées totales $(\mathrm{MAT}=$ azote $\mathrm{Kjeldhal} \times 6,25$ ) et en cellulose brute $(\mathrm{CBw}=$ méthode de Weende) ont été déterminées sur l'aliment, et à chaque stade, après dessication, sur un échantillon moyen représentatif pour les crottes dures et les caecotrophes.

L'exploitation statistique des résultats a été réalisée par analyse de variance ou de covariance à un critère de classification, suivie d'une comparaison des moyennes pour le test de Newman et Keuls. Les équations de prédictions de la quantité de caecotrophes produite ont été calculées par régression multiple et progressive.

\section{Résultats}

\section{A. Quantité et composition des fèces émises}

Etant donné que la pose du collier provoque un stress plus ou moins important, nous n'avons retenu à chaque récolte que les résultats provenant des animaux ayant eu, le jour de la récolte, une excrétion fécale et une ingestion d'aliment équivalentes à celles contrôlées la veille et le lendemain. Ainsi, selon les stades, nous en avons retenu entre 7 et 10 qui ont satisfait à ces conditions, parmi les 10 à 15 mis en essai. Seuls ces animaux ont été pris en considération lors des calculs.

Le poids vif des animaux et leur consommation alimentaire quotidienne s'accroissent pendant toute la durée de l'expérience (tabl. 1). Le gain de poids (GMQ) est maximum entre 8 et 9 semaines (plus de $43 \mathrm{~g} / \mathrm{j}$ ). La production de caecotrophes émise par 24 heures s'accroît rapidement de l'âge de 28 jours jusqu'au moment où est observée la croissance maximum (fig. 1B). Après ce stade, la quantité de caecotrophes excrétée se stabilise entre 20 et $25 \mathrm{~g}$ de MS par jour. La teneur en MS des caecotrophes, élevée au sevrage $(55 \%$ à $28 \mathrm{j})$, diminue ensuite rapidement $(\mathbf{P}<0,01)$ et se stabilise entre $36 \%$ et $40 \%$ (écarts non significatifs entre les moyennes, fig. 1A). Comme dans le cas des caecotrophes, la teneur en MS des fèces dures est élevée au sevrage $(80 \%)$ puis elle diminue $(\mathrm{P}<0,01)$ et se stabilise à environ $60 \%$ après 9 semaines d'âge. La quantité sèche de caecotrophes émises quotidiennement, est équivalente environ au tiers de celle des fèces dures, sauf aux stades 56 et 63 jours (GMQ maximum) où elle équivaut environ à la moitié (fig. 1C). En outre, signalons que l'excrété sec total quotidien (fèces dures + caecotrophes) exprimé en pourcentage de l'ingéré, ne varie pas significativement en fonction de l'âge, bien que les valeurs passent de 48,8 à $57,6 \%$ de 28 à 49 jours d'âge (fig. 1). 
T. GIDENNE, F. LEBAS

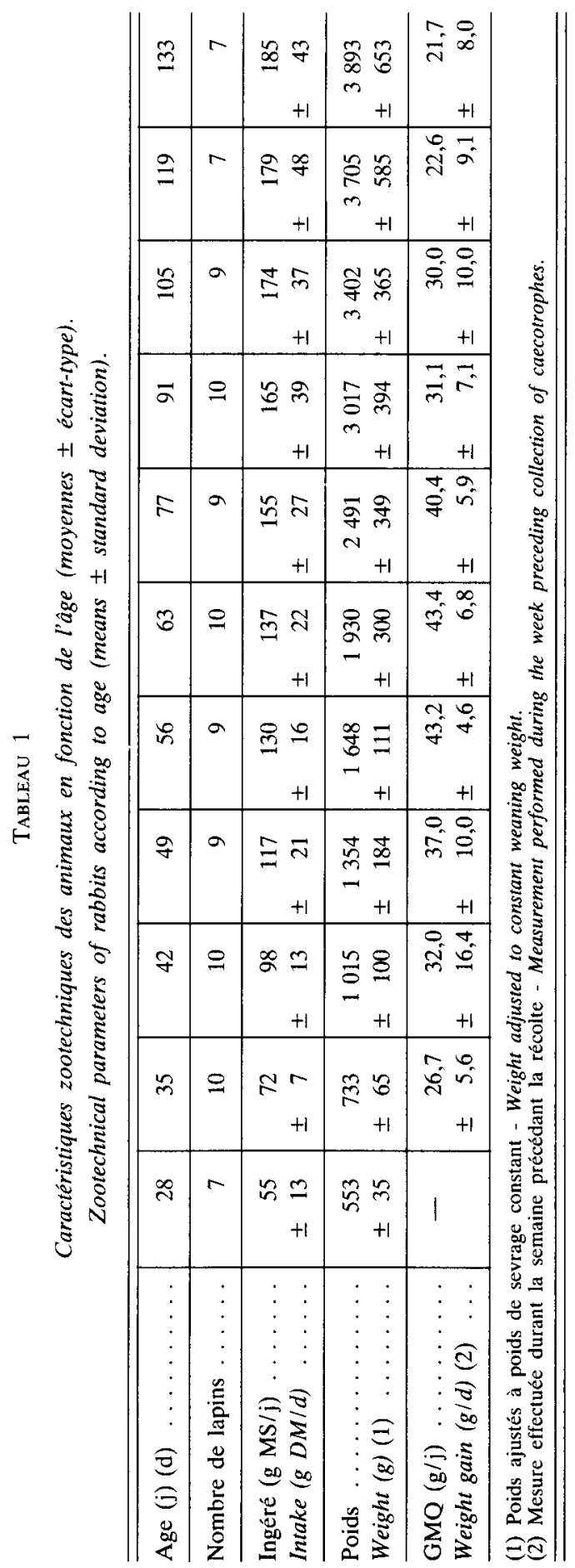




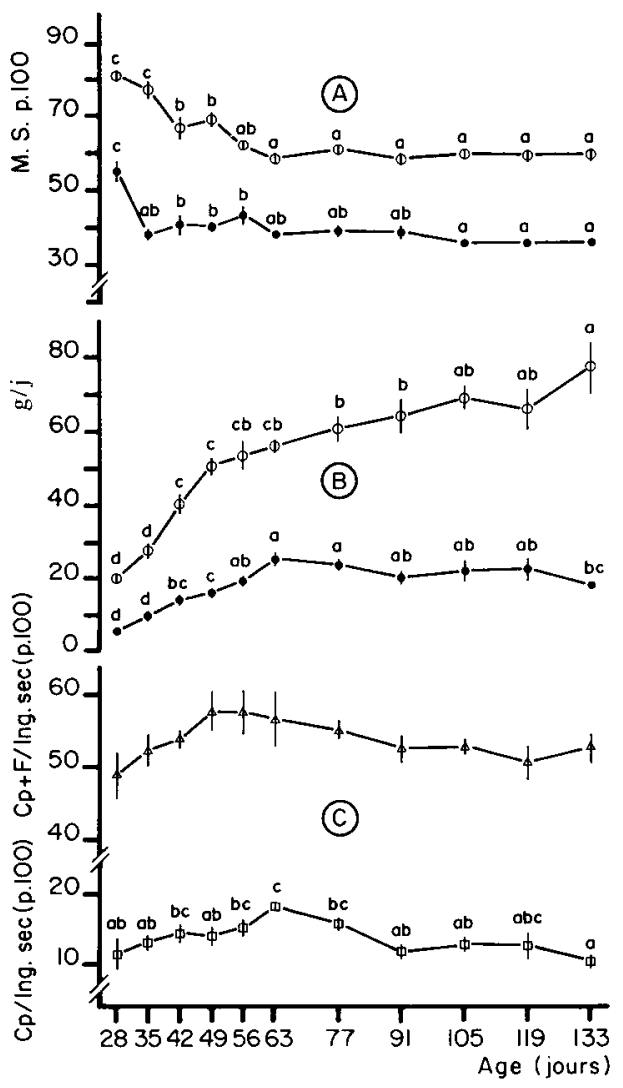

FIG. 1

Evolution avec l'âge de l'excrétion fécale de matière sèche $\bigcirc-\bigcirc$ fèces dures et $\bullet-\bullet$ caecotrophes. Variation with age of the faecal dry matter excretion in the rabbit $\bullet-$ hard faeces -O soft faeces.

A : teneur en matière sèche - dry matter concentration.

B : quantité quotidienne de MS - daily output.

C : quantité de MS totale (caecotrophes + fèces dures) $\triangle-\triangle$ et de MS de caecotrophes $\square-\square$ excrétée en g pour $100 \mathrm{~g}$ de MS d'aliment ingéré.

total dry matter excreted (hard + soft faeces) $\triangle-\Delta$ or as soft faeces $\square-\square$ expressed as g per $100 \mathrm{~g}$ dietary dry matter intake.

Pour une série donnée, deux valeurs affectées d'une même lettre ne sont pas significativement différentes au seuil $\mathrm{P}=0,05$.

For one type of date, values with the same letter are not significantly different at $P=0.05$.

La teneur en MAT des caecotrophes fluctue entre $23 \%$ et $29 \%$ jusqu'à 56 jours d'âge $(29,1 \%)$ (fig. 2) puis elle s'accroît régulièrement de 63 jours $(23,1 \%)$ à 133 jours d'âge $(27,5 \%)$. En moyenne, elle équivaut à plus du double de la teneur en MAT des fèces dures. L'apport d'azote par les caecotrophes, exprimé en pourcentage de l'azote alimentaire ingéré, est élevé en période d'augmentation du GMQ (35 à 77 
jours), excepté à 49 jours d'âge (fig. 2B). En moyenne, sur l'ensemble de la période d'observation, il représente $21,6 \pm 1,0 \%$ de l'azote alimentaire. Il convient aussi de remarquer que l'azote fécal recyclé sous forme de caecotrophes représente une masse équivalente, voire supérieure, à celle éliminée dans les fèces dures. Cet apport alimentaire est le plus important quand les valeurs du GMQ sont maximales, soit entre 56 et 63 jours d'âge. Après l'âge de 11 semaines, cet apport azoté par les caecotrophes est plus faible, il oscille entre 17 et $20 \%$ jusqu'à 133 jours d'âge. Enîin, l'excrétion totale d'azote s'accroît de 28 à 56 jours d'âge, puis diminue régulièrement $(-16,8 \%)$ jusqu'à 133 jours d'âge.

La teneur en cellulose brute des caecotrophes équivaut à environ la moitié de celle des fèces dures, sans variation importante en fonction de l'âge (fig. 3A). Au plan quantitatif, l'apport de cellulose brute par les caecotrophes représente $17,4 \pm 1,1 \%$ de l'apport alimentaire, soit une proportion significativement inférieure $(\mathrm{P}<0,01)$ à celle observée pour l'azote. Cet apport de cellulose brute s'accroît jusqu'à 63 jours d'âge, puis diminue jusqu'au stade 133 jours (fig. 3B).

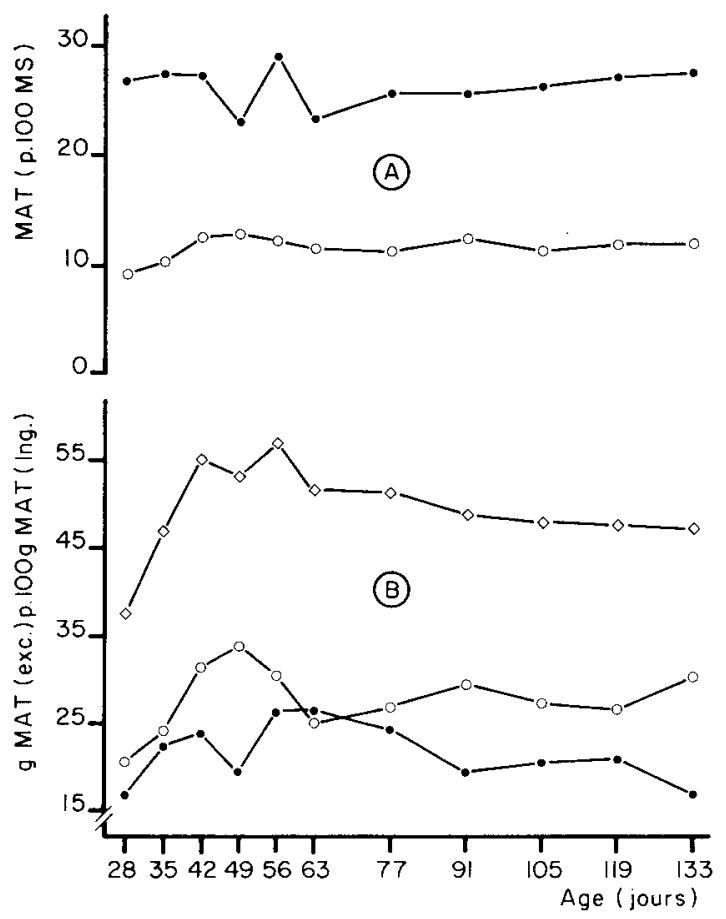

FiG. 2

Caractéristiques de l'excrété azoté moyen en fonction de l'âge.

Characteristics of nitrogen excretion relative to age.

A : teneur en MAT des caecotrophes $\bullet-$ et des fèces dures $\square-0$. crude protein content of soft $\bullet$ and hard faeces $\bigcirc-\circ$.

B : g de MAT excrétée pour $100 \mathrm{~g}$ de MAT alimentaire, dans les caecotrophes $\bullet \bullet \bullet$, les fèces dures $0-0$ et au total $\curvearrowright-0$.

$g$ of fecal crude protein per $100 \mathrm{~g}$ dietary crude protein intake, as soft $\bullet-\bullet$ or hard $\bigcirc-0$ and total faeces $\diamond-\diamond$. 

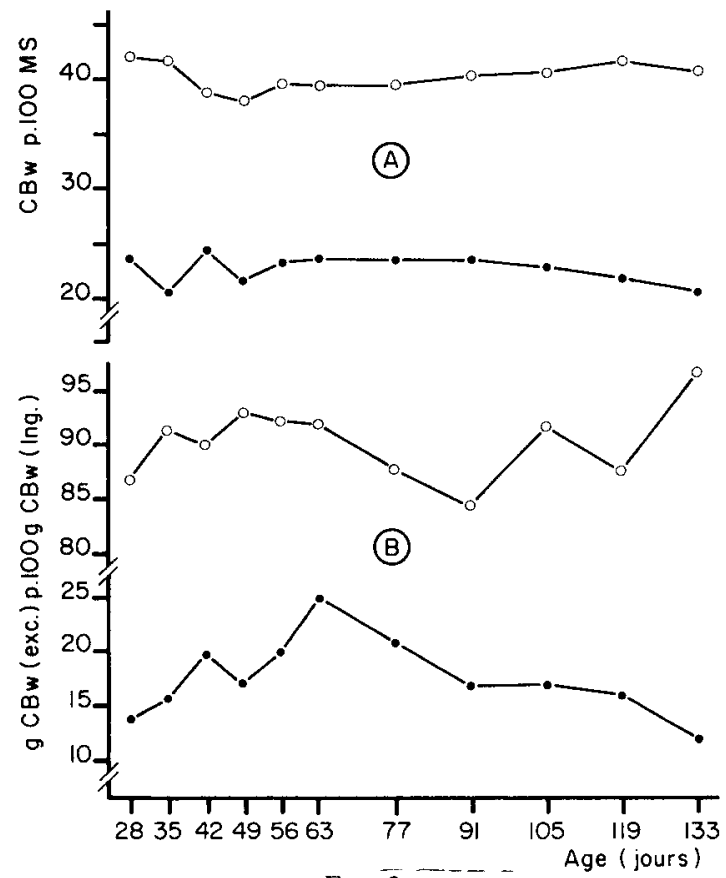

FIG. 3

Caractéristiques de l'excrétion cellulosique (cellulose brute : $C B w$ ) moyenne en fonction de l'âge.

Characteristics of crude fibre ( $C B w)$ excretion relative to age.

A : teneur en $\mathrm{CBw}$ dans les caecotrophes $\bullet$ et les fèces dures $\bigcirc-\mathrm{O}$.

$C B w$ concentration in soft $\bullet-$ and $\bigcirc-0$ hard faeces.

$\mathrm{B}: \mathrm{g}$ de $\mathrm{CBw}$ excrétés, pour $100 \mathrm{~g}$ de $\mathrm{CBw}$ alimentaire, dans les caecotrophes $\bullet-$ et les fèces dures $0-0$.

$g$ of fecal $C B w$ excreted, per $100 \mathrm{~g}$ dietary $C B w$ intake, as soft $\bullet-$ or hard faeces $\bigcirc-\circ$.

\section{B. Estimation de la production de caecotrophes \\ à l'aide de paramètres zootechniques simples}

L'étude par régression multiple progressive, entre 28 et 133 jours d'âge indique que la production de caecotrophes est corrélée à l'ingéré d'aliment et l'excrétion de fèces dures ; l'équation obtenue à partir de 97 mesures est la suivante :

$$
\mathrm{Qcp}=(0,127 \times \mathrm{I})-(0,11 \mathrm{QF})+6,05( \pm 5,3 \mathrm{~g})
$$

avec Qcp = quantité de caecotrophes excrétée $(\mathrm{g} \mathrm{MS} / \mathrm{j})$,

$\mathrm{QF}=$ quantité de fèces dures excrétée $(\mathrm{g} \mathrm{MS} / \mathrm{j})$,

$I=$ consommation d'aliment $(g$ MS $/ \mathrm{j})$.

Le coefficient de corrélation multiple $(\mathrm{R})$ est alors égal à $0,655(\mathrm{P}<0,01)$ et l'écart-type résiduel est de 5,3 grammes.

Les coefficients de corrélation simple avec la production de caecotrophes sont respectivement de 0,639 et 0,509 pour la quantité de fèces dures et la consommation d'aliment. 
L'introduction dans l'équation d'une variable explicative supplémentaire, tel que le poids vif ou le poids métabolique n'augmente pas la précision de l'estimation.

Ainsi, seuls $43 \%$ des variations $\left(\mathrm{R}^{2}\right)$ de la production de caecotrophes sont alors expliqués par I et QF considérés en combinaison. Par ailleurs, notons la forte corrélation entre $Q F$ et $I: r=0,893(P<0,01)$.

La même étude réalisée en période d'élévation de la croissance relative, soit entre 28 et 63 jours, aboutit à l'équation suivante :

$$
\begin{aligned}
& \mathrm{Qcp}=(0,151 \times \mathrm{I})-(0,265 \times \mathrm{QF} / \mathrm{P})+7,93( \pm 4,00): \mathrm{R}=0,827 \\
& \mathrm{QF} / \mathrm{P}=\mathrm{QF} \text { rapportée au poids métabolique de l'individu }\left(\mathrm{P}^{0.75}\right)
\end{aligned}
$$

Ainsi, chez le jeune lapin, à l'aide de l'ingéré et de l'excrétion de fèces dures par unité de poids métabolique, il est possible d'expliquer plus de $68 \%$ des variations de la production de caecotrophes. A l'inverse, la corrélation entre I et $\mathrm{QF}$ est plus faible $(\mathrm{R}=0,786 ; \mathrm{P}<0,01)$ que pour l'ensemble de la période d'observation.

Pour la période 77-133 jours d'âge, nous n'observons plus de corrélation importante entre Qcp et les autres paramètres zootechniques précédents. Le meilleur coefficient de corrélation est mesuré entre Qcp et l'ingéré rapporté au poids métabolique ; il équivaut à 0,437 (19\% des variations de Qcp sont expliquées). Par contre, la corrélation entre $\mathrm{QF}$ et I demeure élevée : $\mathrm{R}=0,882(\mathrm{P}<0,01)$.

\section{Discussion}

La validité des mesures effectuées ne semble pas entachée d'erreur liée au stress éventuel des animaux porteurs du collier, puisque leurs performances de croissance et de consommation sont équivalentes à celles classiquement mesurées. Signalons toutefois que Cantier et al. (1969) observent sur des lapins de souche commune un gain de poids vif maximum entre 5 et 7 semaines d'âge, alors que dans notre cas, il se situe entre 8 et 9 semaines. Cet écart peut s'expliquer d'une part, par des variations interraces (Ouhayoun, 1978), et d'autre part par les conditions d'élevage (cages individuelles...). Un stress éventuel, provoqué peu après le sevrage, par la pose du collier peut également être la cause d'un retard de croissance chez certains animaux.

La caecotrophie est un élément essentiel de la physiologie digestive du lapin. Son intérêt nutritionnel est reconnu, mais on possède peu d'information quant aux facteurs de variations de la quantité de caecotrophes excrétées. Nos résultats portant sur les variations en fonction de l'âge indiquent que le recyclage de matière sèche (MS) par la caecotrophie atteint son niveau le plus élevé au moment où la croissance est aussi la plus forte, soit entre 8 et 11 semaines d'âge. Ainsi l'apport de matière sèche par la caecotrophie ne dépendrait pas simplement de la quantité d'aliment ingérée ; elle ne représente pas non plus une proportion constante de l'excrétion de fèces dures (de 24 à $45 \%$ selon le stade). Ceci suppose une régulation de la caecotrophie au plan quantitatif, qui pourrait être fonction des besoins de l'organisme pour la croissance de l'animal. Ainsi, après 11 semaines d'âge (donc une fois dépassé l'âge d'abattage et la période de forte croissance corporelle, les besoins nutritionnels de l'animal deviennent relativement moins importants, la production de caecotrophes se stabilise alors (faibles corrélations avec le poids ou l'ingéré). Entre 91 et 133 jours d'âge, elle équivaut en moyenne à 
$11,5 \%$ de l'ingéré total sec (aliment et caecotrophes), sans variations significatives selon le stade. Par ailleurs, l'excrétion de fèces dures, exprimée en pourcentage de l'ingéré, ne varie pas significativement en fonction de l'âge : moyenne générale de $40 \mathrm{~g}$ pour $100 \mathrm{~g}$ de MS ingérée.

Lors d'un précédent travail (Gidenne \& Poncet, 1985), la production de caecotrophes a été estimée avec une technique différente (abattage d'animaux nourris avec un aliment contenant un marqueur), ne faisant pas appel à la pose d'un collier. L'estimation était alors de $15 \%$ de l'ingéré total sec, pour des lapins de 8 semaines, recevant un aliment riche en fibres (NDF $=48 \% \mathrm{MS}$ ). Dans le cas présent, au même âge, l'excrété sec de caecotrophes est similaire $(13,0 \%)$, bien que l'aliment soit moins cellulosique (NDF $=34 \% \mathrm{MS}$ ). Chez l'adulte porteur d'un collier, Рroto (1968) notait une excrétion de caecotrophes de $12 \%$ de l'ingéré total de matière sèche, donc équivalente à nos résultats; de plus il n'observait pas de variations importantes en fonction du régime alimentaire (foin ou aliment concentré).

La teneur en MAT des caecotrophes (et leur teneur en eau) équivaut environ au double de celle des fèces, en accord avec les travaux antérieurs (Bonnafous \& Raynaud, 1963 ; Fekete \& Bokori, 1984 ; Furuichi \& Takahashi, 1984). L'importance de la caecotrophie pour la nutrition azotée du lapin a également été montrée en observant une baisse de la digestibilité des protéines chez des lapins porteurs d'un collier (Proto, 1965 ; Stephens, 1977 ; Robinson et al., 1985).

Gidenne \& Poncet (1985) estiment, dans leurs conditions expérimentales, que la caecotrophie permet le recyclage d'une quantité d'azote égale à $20 \%$ de l'ingéré azoté total (aliment + caecotrophes) ; HoRNICKE (1981) pense que le recyclage peut atteindre $30 \%$ de cet ingéré. Nous confirmons ces données puisque l'apport azoté par les caecotrophes atteint $21 \%$ de l'apport total à 8 semaines d'âge, dans notre cas. Selon Falcao e Cunha \& Lebas (1985) ce recyclage de MAT par la caecotrophie ne varie pas suivant la teneur en lignines alimentaires, mais selon la source de fibres. Par ailleurs, ces auteurs observent un recyclage très élevé de MAT, chez des adultes, pouvant atteindre $35 \%$ de l'ingéré azoté total avec certains régimes. JEcsal et al. (1985) trouvent que la caecotrophie influence l'excrétion azotée, non seulement au plan quantitatif, mais aussi au plan qualitatif. Il reste encore à connaitre les variations en fonction de l'âge, de la composition en acides aminés des caecotrophes, plus particulièrement, la fourniture d'acides aminés essentiels par la caecotrophie peut-elle être plus importante en période de forte croissance?

La teneur en cellulose brute que nous mesurons dans les caecotrophes est similaire à celle observée chez l'adulte par Thacker \& Brandt (1955) ou par Proto (1965). Elle varie peu en fonction de l'âge : ainsi, la quantité de cellulose brute recyclée par les caecotrophes est maximum ( $25 \%$ de l'ingéré) lorsque la vitesse de croissance est maximum. Falcao E Cunha \& Lebas (1986) observent chez l'adulte une valeur comparable du recyclage de cellulose brute, sans effet significatif de la teneur en lignines alimentaires.

Le présent travail avait également pour but d'estimer la production de caecotrophes à partir de données simples, mesurables sur des animaux en situation physiologique normale (non porteurs de collier). Nous constatons que, pendant la période 28 à 63 jours d'âge, les variations de la consommation et de l'excrétion de fèces dures (par rapport au poids) expliquent près de $70 \%$ des variations de la quantité de caecotrophes produite. 
Avant que le lapin atteigne 11 semaines d'âge, il semble donc possible de s'affranchir de techniques telles que la pose de collier pour estimer la production de caecotrophes. Toutefois, notre équation n'est basée que sur 56 mesures issues d'un même lot d'animaux relativement homogène. II serait donc utile de renouveler ce type de mesures, en particulier sur un plus grand nombre d'animaux, afin d'améliorer la précision des estimations. De plus, il serait sans doute intéressant de comparer l'excrétion de caecotrophes de lapins nourris avec différents types de régimes, bien que Proto et al. (1968) n'aient pas observé de variations de cette excrétion (en \% de l'ingéré) selon le régime.

Au-delà de l'âge de 77 jours, la situation physiologique de l'animal se stabilise : les animaux ont dépassé la période de forte croissance et, la production quotidienne de caecotrophes ne semble plus varier de manière importante en fonction de l'âge. Nous retiendrons donc, pour le type d'aliment utilisé, que la caecotrophie représente un recyclage quasi-fixe d'environ $20 \mathrm{~g}$ de $\mathrm{MS} / 24$ heures au-delà de 11 semaines d'âge.

En conclusion, au moment où le Lapin présente une croissance élevée, la quantité relative d'azote et de matière sèche recyclée par la caecotrophie atteint son niveau le plus haut. Nous avons observé, avant que le lapin atteigne l'âge habituel d'abattage (11 semaines), une relation étroite entre l'excrétion de caecotrophes et des paramètres aisément mesurables tel que : consommation, excrétion fécale... A l'avenir, il paraît donc possible d'estimer l'excrétion de caecotrophes, en s'affranchissant de techniques relativement lourdes pour l'expérimentateur, ou «stressantes " pour l'animal. Il s'avère également intéressant de préciser au cours de la croissance l'importance de la caecotrophie au plan de la qualité de l'azote et de la nature des constituants pariétaux recyclés.

Reçu en Octobre 1986.

Accepté en Avril 1987.

\section{Remerciements}

Nous remercions A. Lapanousi et $\mathrm{V}$. Sanchez pour leur collaboration technique dans la réalisation de ce travail.

\section{Summary}

Quantitative estimation of caecotrophy in the growing rabbit: variations with age

A study was made to measure caecotrophy in the growing rabbit and to estimate caecotrophy relative to easily measurable performance parameters. Crossbred new Zealand White $\times$ Californian doe rabbits fed ad libitum a complete pelleted diet were kept in digestibility cages from 25 to 133 days of age. Soft faeces excretion was measured for $24 \mathrm{~h}$ every week up to 63 days of age, then every two weeks. At each stage, 7 to 10 animals whose intake and excretion had not been disturbed by the collar preventing caecotrophy were chosen among the 10 to 15 fitted with this collar.

Dry matter content and dry amount of soft faeces stabilized after 11 weeks of age between 36 and $40 \%$, respectively and around $20 \mathrm{~g} \mathrm{DM} /$ day (fig. 1). Total faecal excretion of dry matter (soft + hard faeces) expressed as percent of intake did not change significantly with age (48-55\%). Total crude protein content of soft faeces, twice as large as that of hard faeces changed from 23 to $27 \%$ DM between 63 and 133 days of age and total crude protein supply by soft faeces reached $26 \%$ of dietary protcin supply between 8 and 9 weeks of age. Crude fibre content of soft faeces was $50 \%$ lower than that of hard faeces $(20-24 \%)$ without marked variations according to age.

The results show that reutilization of dry matter and crude protein was maximum during the period with the highest growth rate (figure $1 \mathrm{C}$ ). From 28 to 63 days of age soft faeces excretion 
was correlated with a linear combination between intake and soft faeces excretion $(\mathrm{R}=0.827$; $\mathbf{P}<0.01)$. Consequently, before slaughter age (11 weeks) and with our experimental diet, it seems possible to estimate quantitatively soft faeces excretion from performance parameters without using a collar.

Key words: Rabbit, caecotrophy, effect of age, equations of prediction.

\section{Références bibliographiques}

Battaglini M.B., 1968. Importanza della coprophagia nil coniglio domestico, in rapporto alla utilissazione de alcuni principi nutritivi. Riv. Zootec. Agric. Vet., 6, 21-37.

Bonnafous R., Raynaud P., 1963. Recherches sur le rythme nycthéméral de l'excrétion de l'azote fécal chez le lapin domestique (Oryctolagus cuniculi). Influence des surrénales. Comp. Biochem. Physiol., 8, 321-326.

Cantier J., Vezinhet A., Rouvier R., Dauzier L., 1969. Allométrie de croissance chez le lapin. I. Principaux organes et tissus. Ann. Biol. anim. Bioch. Biophys., 14, 271-292.

Catala J., 1976. Etudes sur les répartitions hydrique, pondérale et azotée dans le matériel digestif chez le lapin, en relation avec la dualité de l'excrétion fécale. $1^{e r}$ Congrès International Cunicole, Dijon, communication $\mathrm{n}^{\circ} 58$.

Falcao e Cunha Luisa, Lebas F., 1986. Influence chez le lapin adulte de l'origine et du taux de lignine alimentaire sur la digestibilité de la ration et l'importance de la caecotrophie. $4^{e s}$ Journées de la Recherche Cunicole en France, communication 8.

Fekete S., Bokori J., 1985. The effect of the fiber and protein level of the ration upon the caecotrophy of rabbit. J. appl. Rabbit Res., 8, 68-71.

Furuichi Y., Takahashi T., 1984. Movements of water soluble and insoluble markers in the digestive tract of rabbits. Jap. J. Zootech. Sci., 55, 552-561.

Gallouin F., 1981. Intêrêt nutritionnel et déterminisme de la caecotrophie chez le lapin. Thèse d'Etat, Université Paris VI.

Gidenne T., Lebas F., 1984. Evolution circadienne du contenu digestif chez le lapin en croissance. Relation avec la caecotrophie. $3^{\text {p }}$ Congrès Mondial de Cuniculture, Rome, Vol. 2, $494-501$.

Gidenne T., Poncet C., 1985. Digestion chez le lapin en croissance d'une ration à taux élevé de constituants pariétaux : étude méthodologique pour le calcul de digestibilité apparente, par segment digestif. Ann. Zootech., 34, 429-446.

Hornicke H., 1981. Utilization of cecal digesta by caecotrophy (soft faeces ingestion) in the rabbit. Livest. Prod. Sci., 8, 361-366.

Jecsai J., Teleki M., Juhasz B., 1985. Effect of caecotrophy on protein and aminoacid metabolism of Angora rabbits. Acta. Vet. Hung., 33, 51-57.

JILGE B., 1974. Soft faeces excretion and passage time in the laboratory animals. Lab. anim., 8, 337-346.

Kulwich R., Struglia L., Pearson P.B., 1953. The effect of coprophagy on the excretion of B vitamine by the rabbit. J. Nutr., 49, 639-645.

Ouhayoun J., 1978. Etude comparative de races de lapins différant par le poids adulte. Thèse $3^{\mathrm{e}}$ Cycle, Université de Montpellier.

Piekarz R., 1963. Effet de la coprophagie sur le temps de transit digestif chez le lapin domestique. Acta. physiol. polon., 14, 359-370.

Proto V., 1965. Experienze di coprogagia nel coniglio. Prod. Anim., 4, 1-21.

Proto V., Gargano D., Gianani L., 1968. La coprofagia del coniglio sottoposto a differenti diete. Prod. Anim., 7, 157-171.

Robinson K.L., Cheeke P.R., Patton N.M., 1985. Effect of prevention of coprophagy on the digestibility of high forage and high concentrate diets by rabbits. J. appl. Rabbit Res., 8, 5759.

Stephens A.G., 1977. Digestibility and coprophagy in the growing rabbits. Proc. Nutr. Soc., 36 , $4 \mathrm{~A}$.

Thacker E.J., Brandt C.S., 1955. Coprophagy in the rabbit. J. Nutr., 55, 375-385. 